\title{
Growth and Yield of Different Size-Seedling Tubers Derived from True Potato (Solanum tuberosum L.) Seeds as Influenced by Clump Planting
}

\author{
R. N. Rojoni ${ }^{1}$, T. S. Roy ${ }^{2}$, M. D. Sarkar ${ }^{1}$, K. Kabir ${ }^{1}$ and A. Ullah ${ }^{1}$ \\ ${ }^{1}$ Department of Horticulture, ${ }^{2}$ Department of Agronomy, Sher-e-Bangla Agricultural University, \\ Dhaka-1207, Bangladesh \\ *Corresponding author and Email: dulalsau_121@yahoo.com
}

Received: 11 February $2014 \quad$ Accepted: 14 June 2014

\begin{abstract}
An experiment was conducted at the Horticulture farm of Sher-e-Bangla Agricultural University, Dhaka, Bangladesh during November 2010 to March 2011. Four levels of seedling tuber size, viz. 6-<7 $\mathrm{g}\left(\mathrm{S}_{1}\right), 7-<8 \mathrm{~g}\left(\mathrm{~S}_{2}\right), 8-<9 \mathrm{~g}\left(\mathrm{~S}_{3}\right)$ and, 9- $\leq 10 \mathrm{~g}\left(\mathrm{~S}_{4}\right)$ and three levels of seedling tuber, viz. 1 hill $^{-1}\left(\mathrm{~N}_{1}\right), 2$ hill $^{-1}\left(\mathrm{~N}_{2}\right)$ and 3 hill $^{-1}\left(\mathrm{~N}_{3}\right)$ were laid out in a Randomized Complete Block Design with three replications. At harvest, the tallest plant $(82.17 \mathrm{~cm})$, highest number of leaves plant ${ }^{-1}(83.30)$, highest LAI (6.23), tubers weight hill ${ }^{-1}(128.90 \mathrm{~g})$ and tuber yield ha ${ }^{-1}(25.78 \mathrm{t})$ were found in $\mathrm{S}_{4}$, while the lowest was in $\mathrm{S}_{1}$. On the other hand, the tallest plant $(74.91 \mathrm{~cm})$, highest number of leaves plant ${ }^{-1}$ (74.45), highest LAI (5.41), tubers weight $\operatorname{hill}^{-1}(123.95 \mathrm{~g})$ and tuber yield ha ${ }^{-1}(24.79 \mathrm{t})$ were found in $\mathrm{N}_{3}$, while the lowest was in $\mathrm{N}_{1}$. The tallest plant $(85.09 \mathrm{~cm})$, highest number of leaves plant ${ }^{-1}(87.97)$, highest LAI (6.64), tubers weight hill ${ }^{-1}(138.35 \mathrm{~g})$ and tuber yield ha ${ }^{-1}(27.67 \mathrm{t})$ were found in $\mathrm{S}_{4} \mathrm{~N}_{3}$ and the lowest gross tuber yield $h^{-1}(12.83 \mathrm{t})$ was from $\mathrm{S}_{1} \mathrm{~N}_{1}$. Large seedling tuber with single seedling tuber hill ${ }^{-1}$ performed better.
\end{abstract}

Keywords: Seedling tuber, clump planting, morphological features, potato yield.

\section{Introduction}

In Bangladesh, potato (Solanum tuberosum L.) is one of the major crops next to rice and wheat and it covers an area of about 403.4 thousand hectares of land yielding $14.74 \mathrm{t} \mathrm{ha}^{-1}$ (MOA, 2009). The national average yield of potato in Bangladesh is much lower compared to many potato growing countries of the world like Belgium, the Netherlands, UK, Germany and USA where the average yield ranges between 38.4 to $49.0 \mathrm{t} \mathrm{ha}^{-1}$ (FAO, 1999). This low yield of potato in Bangladesh are due to lack of quality seed tuber, environmental limitations, unavailability and uneven distribution of certified seeds and use of indigenous cultivars
(Roy et al., 2009; Roy et al., 2005).Potato production in Bangladesh may be increased by improving cultural practices which are optimization of manure and fertilizer, planting time, spacing and use of optimal size seed tubers which influences the yield of potato (Divis and Barta, 2001). Development of true potato seed (TPS) technology has opened a new era in potato cultivation. TPS progeny may produce 500 to 800 seedling tubers $\mathrm{m}^{-2}$ of land when planted at $10 \mathrm{~cm} \times 10 \mathrm{~cm}$ spacing (TCRC, 2004). Small seedling tubers $(\approx 10 \mathrm{~g})$ are usually neglected In Bangladesh both in terms of ware potato and seed tuber, although the genetical constitution of small seedling tuber is more or less similar to that of standard one (Upadhya et al., 2003). 
Seedling tubers may be planted together in a hill, which is known as clump planting and could behave equally to single bigger seed tuber. Seedling tuber size and clump planting may be considered as very important factors for the production of potato. In traditional method of potato production, seedling tuber size and plant population per hill have been found to influence the yield and economic return (Hossain, 2004). Considering the importance of size of seedling tubers and clump planting, the present study was undertaken to study the growth and yield performance and develop a feasible standard method for utilization of TPS seedling tubers.

\section{Materials and Methods}

\subsection{Experimental site, soil and climate}

The experiment was conducted at the Horticulture Farm of Sher-e-Bangla Agricultural University, Dhaka-1207, Bangladesh during November 2010 to March 2011.Soil of the experimental field was a silty loam in texture under AEZ Madhupur Tract.

\subsection{Plating material}

TPS seedling tubers of the variety BARI TPS-1 were used in this experiment which is collected from the Tuber Research Centre, Bangladesh Agriculture Research Institute, Gazipur. The variety is a hybrid between female parent MF-II and male parent TPS-67 that was released by the National Seed Board (NSB) during 1997.

\subsection{Treatments and design of the experiment}

The experiment consisted of two factors viz., seedling tuber size and clump planting. Clump planting is the technique of planting of more than one tuber hill $^{-1}$ and the plant hill ${ }^{-1}$ counting as single plant. Factor A: Four levels of seedling tuber size, viz. 6-<7 g $\left(\mathrm{S}_{1}\right), 7-<8 \mathrm{~g}\left(\mathrm{~S}_{2}\right), 8-<9 \mathrm{~g}$ $\left(\mathrm{S}_{3}\right)$ and, $9-\leq 10 \mathrm{~g}\left(\mathrm{~S}_{4}\right)$ and three levels of seedling tuber, viz. 1 hill $^{-1}\left(\mathrm{~N}_{1}\right), 2$ hill $^{-1}\left(\mathrm{~N}_{2}\right)$ and 3 hill $^{-1}\left(\mathrm{~N}_{3}\right)$ were tested in this study. The experiment was laid out in a Randomized Complete Block Design with three replications. The total area of the experimental plot was divided into three equal blocks. Each block was divided into 12 plots and there were 36 unit plots altogether. The size of the each plot was $1 \mathrm{~m} \times 1$ $\mathrm{m}$. The distances between rows was $50 \mathrm{~cm}$ and between plants was $10 \mathrm{~cm}$.

\subsection{Land preparation and application of manure and fertilizers}

The field was opened in the $2^{\text {nd }}$ week of November 2010 with a power tiller and left exposed to the sun for a week. The fertilizers were applied at 300, 220, 250, 100, 25, 15 and $10000 \mathrm{kgha}^{-1}$ as urea, TSP, MP, gypsum, Zinc sulphate, boric acid and cowdung, respectively (TCRC, 2004). Fifty percent of urea and full amount of other fertilizers were applied during final land preparation. Moreover, Carbofuran 5G @ $15 \mathrm{~kg} \mathrm{ha}^{-1}$ was also applied during that time.

\subsection{Seed preparation, sowing and intercultural operation}

The tubers were graded according to the size and kept under diffuse light conditions to have healthy and good sprouts. Planting was done on November 28, 2010.Intercultural operations like weeding, earthing-up, irrigation were furnished for proper growth and development of the crop. Dithane M-45 was sprayed in two instalment at an interval of 15 days from 50 DAP as preventive measure against late blight disease.

\subsection{Harvesting, data collection and statistical analysis}

The crop was harvested at 100 DAP when the plants showed 80 to $90 \%$ of leaf senescence. Haulm cutting was done before 7 days of harvesting. Ten plants were randomly selected from each unit plot and data on days to emergence, plant height, number of leaves, leaf area index, fresh mass, dry mass, crop growth rate, weight of tuber hill $^{-1}$ and yield of tubers were collected. Leaf area index (LAI) and crop growth rate (CGR) were computed following standard formula as given below:

$$
\text { LAI }=\frac{\text { Leaf area }}{\text { Unit land area }}
$$

Where,

Unit land area $=$ Spacing of plant to plant and row to row $(10 \times 50 \mathrm{~cm})$ 


$$
\mathrm{CGR}=\frac{\mathrm{W}_{2}-\mathrm{W}_{1}}{-------} \mathrm{g} \mathrm{m}^{-2} \mathrm{day}^{-1}
$$

Where,

$\mathrm{W}_{2}$ and $\mathrm{W}_{1}$ are the dry mass at time $\mathrm{T}_{2}$ and $\mathrm{T}_{1}$, respectively.

Data were analysed with the help of MSTAT-C Program and mean values of all the parameters were adjudged by least significance difference (LSD) at 5\% level of probability.

\section{Results and Discussion}

\subsection{Days to first emergence}

The emergence was influenced significantly due to seedling tubers size. Seedling tubers $(8-<9 \mathrm{~g}$ and $9-\leq 10 \mathrm{~g}$ ) required less duration (7.56 days), whereas seedling tubers $(7-<8 \mathrm{~g})$ required comparatively higher duration (8.67 days) for first emergence but it was statistically similar to 6-<7 g seedling tubers (Table 1).The duration required for first emergence was not influenced significantly by the clump planting (Table 1) and also by the treatment combination of seedling tuber size and clump planting (Table 2).

\subsection{Days to $80 \%$ emergence}

Days to $80 \%$ emergence was influenced significantly due to seedling tuber size (Table 1). Seedling tubers $(8-<9 \mathrm{~g}$ and $9-\leq 10 \mathrm{~g})$ required less duration (10.78 days), whereas seedling tubers $(6-<7 \mathrm{~g})$ required higher duration $(12.33$ days). The results indicate that duration required for emergence decreased gradually with the increase in tuber size. The early emergence from large seedling tubers was probably due to more reserve food material. This result is in agreement with the findings of Hossain (2004) who reported that emergence was faster in case of larger tubers compared to smaller ones. Emergence of plants was also influenced significantly by the clump planting. Minimum duration (10.92 days) was required in $\mathrm{N}_{3}$ compared to 1 or 2 seedling tubers hill ${ }^{-1}$ (Table 1).Significant variation was recorded among the treatment combinations. Minimum duration for $80 \%$ emergence of plants required in $\mathrm{S}_{4} \mathrm{~N}_{3}$ (Table 2). This result is in agreement with the findings of Hossain (2004) who reported that emergence was faster in case of larger seedling tubers with higher number of seedling tubers hill $^{-1}$.

Table 1. Effect of seedling tuber size and clump planting on the emergence of plant and plant height at different days after planting (DAP)

\begin{tabular}{|c|c|c|c|c|c|c|}
\hline \multirow{2}{*}{ Treatments } & \multirow{2}{*}{$\begin{array}{l}\text { Days to } 1^{\text {st }} \\
\text { emergence }\end{array}$} & \multirow{2}{*}{$\begin{array}{c}\text { Days to } 80 \% \\
\text { emergence }\end{array}$} & \multicolumn{4}{|c|}{ Plant height $(\mathrm{cm})$} \\
\hline & & & 40 DAP & 55 DAP & 70 DAP & 100 DAP \\
\hline \multicolumn{7}{|c|}{ Seedling tuber size } \\
\hline $\mathrm{S}_{1}$ & $8.11 \mathrm{ab}$ & $12.33 \mathrm{a}$ & $39.14 \mathrm{~d}$ & $47.12 \mathrm{~d}$ & $55.49 \mathrm{~d}$ & $65.99 \mathrm{~d}$ \\
\hline $\mathrm{S}_{2}$ & $8.67 \mathrm{a}$ & $11.56 \mathrm{ab}$ & $44.48 \mathrm{c}$ & $51.69 \mathrm{c}$ & $60.48 \mathrm{c}$ & $69.46 \mathrm{c}$ \\
\hline $\mathrm{S}_{3}$ & $7.56 \mathrm{~b}$ & $11.11 \mathrm{~b}$ & $50.47 \mathrm{~b}$ & $58.38 \mathrm{~b}$ & $67.23 \mathrm{~b}$ & $74.37 \mathrm{~b}$ \\
\hline $\mathrm{S}_{4}$ & $7.56 \mathrm{~b}$ & $10.78 \mathrm{~b}$ & $55.51 \mathrm{a}$ & $65.00 \mathrm{a}$ & $72.84 \mathrm{a}$ & $82.17 \mathrm{a}$ \\
\hline $\mathrm{LSD}_{(0.05)}$ & 0.81 & 0.89 & 1.33 & 1.57 & 1.19 & 0.87 \\
\hline \multicolumn{7}{|c|}{ Seedling tuber hill $^{-1}$} \\
\hline $\mathrm{N}_{1}$ & 8.00 & $12.00 \mathrm{a}$ & $46.07 \mathrm{~b}$ & $54.23 \mathrm{~b}$ & $62.78 \mathrm{~b}$ & $72.10 \mathrm{~b}$ \\
\hline $\mathrm{N}_{2}$ & 8.08 & $11.42 \mathrm{ab}$ & $47.17 \mathrm{~b}$ & $54.89 \mathrm{~b}$ & $63.06 \mathrm{~b}$ & $71.98 \mathrm{~b}$ \\
\hline $\mathrm{N}_{3}$ & 7.83 & $10.92 \mathrm{~b}$ & $48.96 \mathrm{a}$ & $57.52 \mathrm{a}$ & $66.19 \mathrm{a}$ & $74.91 \mathrm{a}$ \\
\hline $\operatorname{LSD}_{(0.05)}$ & 0.71 & 0.78 & 1.15 & 1.36 & 1.03 & 0.76 \\
\hline
\end{tabular}

In a column, means followed by same letter (s) do not differ significantly at $5 \%$ level of probability, $\mathrm{S}_{1}=6-<7 \mathrm{~g}, \mathrm{~S}_{2}=$ $7-<8 \mathrm{~g}, \mathrm{~S}_{3}=8-<9 \mathrm{~g}, \mathrm{~S}_{4}=9-\leq 10 \mathrm{~g}, \mathrm{~N}_{1}=1$ seedling tuber hill ${ }^{-1}, \mathrm{~N}_{2}=2$ seedling tubers hill ${ }^{-1}$ and $\mathrm{N}_{3}=3$ seedling tubers hill $^{-1}$ 
Table 2. Combined effect of seedling tuber size and clump planting on the emergence of plant and plant height at different days after planting (DAP)

\begin{tabular}{ccccccc}
\hline \multirow{2}{*}{ Treatments } & Days to $1^{\text {st }}$ & Days to 80\% & \multicolumn{4}{c}{ Plant height $(\mathrm{cm})$} \\
\cline { 4 - 7 } & emergence & emergence & 40 DAP & 55 DAP & 70 DAP & 100 DAP \\
\hline $\mathrm{S}_{1} \mathrm{~N}_{1}$ & 8.33 & $13.00 \mathrm{ab}$ & $37.87 \mathrm{i}$ & 47.00 & 55.03 & $66.01 \mathrm{i}$ \\
$\mathrm{S}_{1} \mathrm{~N}_{2}$ & 8.00 & $13.00 \mathrm{ab}$ & $38.80 \mathrm{hi}$ & 46.27 & 54.13 & $64.30 \mathrm{j}$ \\
$\mathrm{S}_{1} \mathrm{~N}_{3}$ & 8.00 & $11.00 \mathrm{c}$ & $40.75 \mathrm{gh}$ & 48.10 & 57.32 & $67.67 \mathrm{~h}$ \\
$\mathrm{~S}_{2} \mathrm{~N}_{1}$ & 9.33 & $13.33 \mathrm{a}$ & $44.40 \mathrm{ef}$ & 51.47 & 59.97 & $69.35 \mathrm{fg}$ \\
$\mathrm{S}_{2} \mathrm{~N}_{2}$ & 8.67 & $10.67 \mathrm{c}$ & $43.00 \mathrm{fg}$ & 50.40 & 59.03 & $68.66 \mathrm{gh}$ \\
$\mathrm{S}_{2} \mathrm{~N}_{3}$ & 8.00 & $10.67 \mathrm{c}$ & $46.03 \mathrm{de}$ & 53.20 & 62.45 & $70.36 \mathrm{f}$ \\
$\mathrm{S}_{3} \mathrm{~N}_{1}$ & 7.33 & $11.00 \mathrm{c}$ & $47.53 \mathrm{~d}$ & 56.10 & 65.53 & $73.20 \mathrm{e}$ \\
$\mathrm{S}_{3} \mathrm{~N}_{2}$ & 8.00 & $10.67 \mathrm{c}$ & $50.95 \mathrm{c}$ & 57.97 & 67.25 & $73.40 \mathrm{e}$ \\
$\mathrm{S}_{3} \mathrm{~N}_{3}$ & 7.33 & $11.67 \mathrm{bc}$ & $52.93 \mathrm{bc}$ & 61.07 & 68.90 & $76.52 \mathrm{~d}$ \\
$\mathrm{~S}_{4} \mathrm{~N}_{1}$ & 7.00 & $10.67 \mathrm{c}$ & $54.47 \mathrm{ab}$ & 62.37 & 70.57 & $79.85 \mathrm{c}$ \\
$\mathrm{S}_{4} \mathrm{~N}_{2}$ & 7.67 & $11.33 \mathrm{c}$ & $55.93 \mathrm{a}$ & 64.93 & 71.83 & $81.57 \mathrm{~b}$ \\
$\mathrm{~S}_{4} \mathrm{~N}_{3}$ & 8.00 & $10.33 \mathrm{c}$ & $56.13 \mathrm{a}$ & 67.70 & 76.11 & $85.09 \mathrm{a}$ \\
\hline $\mathrm{LSD}_{(0.05)}$ & 1.41 & 1.56 & 2.30 & 2.73 & 2.06 & 1.51 \\
\hline
\end{tabular}

In a column, means followed by same letter (s) do not differ significantly at $5 \%$ level of probability, $\mathrm{S}_{1}=6-<7 \mathrm{~g}$, $\mathrm{S}_{2}=7-<8 \mathrm{~g}, \mathrm{~S}_{3}=8-<9 \mathrm{~g}, \mathrm{~S}_{4}=9-\leq 10 \mathrm{~g}, \mathrm{~N}_{1}=1$ seedling tuber hill ${ }^{-1}, \mathrm{~N}_{2}=2$ seedling tubers hill $^{-1}$ and $\mathrm{N}_{3}=3$ seedling tubers hill ${ }^{-1}$

\subsection{Plant height}

Plant height was significantly influenced by seedling tuber size at 40,55, 70 and 100 DAP of potato (Table 1). Increased size of seedling tuber significantly increased the plant height. The tallest plant at 40, 55, 70 and 100 DAP were recorded in $\mathrm{S}_{4}$. Whereas, the shortest plants were at $40,55,70$ and 100 in $S_{1}$. The plant height was higher in larger seedling tubers because larger seedling tubers had huge stored food material that supported increased vegetative growth of the plants. This result is consistent with several other findings (Garget al., 2000; Reust, 2002; Hossain, 2004; Tohin, 2010) in potato they reported that plant height of potato increased with increasing seed tuber size.

Plant height was influenced significantly by the clump planting. The tallest plants at $40,55,70$ and 100 DAP were found in $\mathrm{N}_{3}$. Whereas, the shortest plants at 40, 55 and 70 DAP were found in $\mathrm{N}_{1}$, while the shortest plants at 100 DAP were from $\mathrm{N}_{2}$ and it was statistically similar to $\mathrm{N}_{1}$. The highest plants grown from 3 seedling tubers hill ${ }^{-1}$ was probably due to more inter-plant competition for sunlight (Table 1). This result is in agreement with the findings of Hossain (2004). The plant height was influenced significantly by the treatment combinations at 40 and 100 DAP (Table 2). The tallest plants at 40 and 100 DAP were recorded in $\mathrm{S}_{4} \mathrm{~N}_{3}$. On the other hand, the shortest plants at 40 DAP were from $\mathrm{S}_{1} \mathrm{~N}_{1}$ and at $100 \mathrm{DAP}$ from $\mathrm{S}_{1} \mathrm{~N}_{2}$.

\subsection{Number of leaves per plant}

The foliage coverage of plants was influenced significantly at all dates of observations due to seedling tuber size. The highest number of leaves plant $^{-1}$ at all dates of observations was found in $\mathrm{S}_{4}$, while the lowest number of leaves plant ${ }^{-1}$ was found in $S_{1}$. The result revealed that the number of leaves increased gradually with the increase in seedling tuber size (Table 3). This trend of the present results agreed with that of Gulluoglu and Aroglu (2009) and Hossain (2004) in potato. They were reported that leaf number in potato decreased with decreasing tuber weight. 
Clump planting significantly influenced the number of leaves plant ${ }^{-1}$. The maximum number of leaves plant ${ }^{-1}$ at all dates of observation was recorded in $\mathrm{N}_{3}$. On the other hand, the minimum number of leaves plant $^{-1}$ at all dates of observations was found $\mathrm{inN}_{1}$. The results indicated that the number of leaves plant ${ }^{-1}$ were directly proportional to the clump planting (Table 3). This result is in agreement with the findings of Hossain (2004). There were significant variations among the treatment combinations of seedling tubers size and clump planting. The highest number of leaves plant ${ }^{-1}$ at all dates of observations were found in $\mathrm{S}_{4} \mathrm{~N}_{1}$ whereas, the lowest number of leaves plant ${ }^{-1}$ at 40 DAP was from $S_{1} \mathrm{~N}_{2}$. At 55 and 70 DAP, the lowest number of leaves plant $^{-1}$ were recorded from 6-<7 g seedling tuber size with 1 and 2 seedling tubers hill $^{-1}$, respectively while at 100 DAP, the lowest number of leaves plant ${ }^{-1}$ was found in $\mathrm{S}_{1} \mathrm{~N}_{2}$ (Table 4). This result is in agreement with that of Hossain (2004).

\subsection{Leaf area index}

The effect of seedling tuber size on leaf area index (LAI) was significant at 40, 55, 70 and 100
DAP (Table 3). LAI increased with increasing tuber size. The highest LAI was recorded in 9$\leq 10 \mathrm{~g}$ seedling tuber at all dates of observations. The lowest LAI was recorded in 6- $<7 \mathrm{~g}$ seedling tuber at 40, 55, 70 and 100 DAP were recorded in $6-<7 \mathrm{~g}$ seedling tuber (Table 5). This result is supported by Verma et al., (2007) in potato.

The LAI was significantly influenced by clump planting at 40, 55, 70 and 100 DAP (Table 3). LAI increased with increasing number of seedling tuber hill $^{-1}$. The highest LAI was recorded in $\mathrm{N}_{3}$ while the lowest was recorded in $\mathrm{N}_{1}$. The interaction effect of seedling tuber size and clump planting was significant on LAI at 70 and 100 DAP except at 40 and 55 DAP (Table 4). The highest LAI was recorded in $\mathrm{S}_{4} \mathrm{~N}_{3}$ at 70 and 100 DAP.

\subsection{Fresh mass production per plant}

Fresh mass production plant ${ }^{-1}$ showed significant variations due to seedling tuber size. The highest fresh mass plant ${ }^{-1}$ at 40, 55, 70 and 100 DAP were recorded in $\mathrm{S}_{4}$. The lowest fresh mass plant ${ }^{1}$ at all dates of observations was recorded in $S_{1}$ (Table 5).

Table 3. Effect of seedling tuber size and clump planting on the number of leaves hill $^{-1}$ and leaf area index at different days after planting

\begin{tabular}{|c|c|c|c|c|c|c|c|c|}
\hline \multirow{2}{*}{ Treatments } & \multicolumn{4}{|c|}{ Number of leaves hill ${ }^{-1}$} & \multicolumn{4}{|c|}{ Leaf area index } \\
\hline & 40 DAP & 55 DAP & 70 DAP & 100 DAP & 40 DAP & 55 DAP & 70 DAP & 100 DAP \\
\hline \multicolumn{9}{|c|}{ Seedling tuber size } \\
\hline $\mathrm{S}_{1}$ & $28.12 \mathrm{~d}$ & $36.32 \mathrm{~d}$ & $50.26 \mathrm{~d}$ & $62.84 \mathrm{~d}$ & $2.42 \mathrm{~d}$ & $2.72 \mathrm{~d}$ & $3.77 \mathrm{~d}$ & $4.30 \mathrm{~d}$ \\
\hline $\mathrm{S}_{2}$ & $35.10 \mathrm{c}$ & $44.58 \mathrm{c}$ & $56.43 \mathrm{c}$ & $68.29 \mathrm{c}$ & $2.89 \mathrm{c}$ & $3.15 \mathrm{c}$ & $4.07 \mathrm{c}$ & $4.54 \mathrm{c}$ \\
\hline $\mathrm{S}_{3}$ & $41.40 \mathrm{~b}$ & $49.09 \mathrm{~b}$ & $61.74 \mathrm{~b}$ & $74.48 \mathrm{~b}$ & $3.61 \mathrm{~b}$ & $4.00 \mathrm{~b}$ & $4.97 \mathrm{~b}$ & $5.45 \mathrm{~b}$ \\
\hline $\mathrm{S}_{4}$ & $46.87 \mathrm{a}$ & $55.68 \mathrm{a}$ & $69.11 \mathrm{a}$ & $83.30 \mathrm{a}$ & $4.16 \mathrm{a}$ & $4.8 \mathrm{a}$ & $5.85 \mathrm{a}$ & $6.23 \mathrm{a}$ \\
\hline $\operatorname{LSD}_{(0.05)}$ & 1.52 & 1.67 & 1.32 & 1.19 & 0.11 & 0.50 & 0.22 & 0.23 \\
\hline \multicolumn{9}{|c|}{ Seedling tuber hill' ${ }^{-1}$} \\
\hline $\mathrm{N}_{1}$ & $36.56 \mathrm{~b}$ & $43.56 \mathrm{c}$ & $57.54 \mathrm{c}$ & $70.61 \mathrm{~b}$ & $3.10 \mathrm{c}$ & $3.32 \mathrm{~b}$ & $4.43 \mathrm{~b}$ & $4.97 \mathrm{~b}$ \\
\hline $\mathrm{N}_{2}$ & $37.62 \mathrm{~b}$ & $46.69 \mathrm{~b}$ & $59.20 \mathrm{~b}$ & $71.63 \mathrm{~b}$ & $3.21 \mathrm{~b}$ & $3.61 \mathrm{ab}$ & $4.62 \mathrm{~b}$ & $5.00 \mathrm{~b}$ \\
\hline $\mathrm{N}_{3}$ & $39.44 \mathrm{a}$ & $49.00 \mathrm{a}$ & $61.41 \mathrm{a}$ & $74.45 \mathrm{a}$ & $3.49 \mathrm{a}$ & $3.99 \mathrm{a}$ & $4.93 \mathrm{a}$ & $5.41 \mathrm{a}$ \\
\hline $\operatorname{LSD}_{(0.05)}$ & 1.32 & 1.45 & 1.14 & 1.03 & 0.09 & 0.43 & 0.19 & 0.20 \\
\hline
\end{tabular}

In a column, means followed by same letter (s) do not differ significantly at $5 \%$ level of probability, $\mathrm{S}_{1}=6-<7 \mathrm{~g}$, $\mathrm{S}_{2}=7-<8 \mathrm{~g}, \mathrm{~S}_{3}=8-<9 \mathrm{~g}, \mathrm{~S}_{4}=9-\leq 10 \mathrm{~g}, \mathrm{~N}_{1}=1$ seedling tuber hill ${ }^{-1}, \mathrm{~N}_{2}=2$ seedling tubers hill ${ }^{-1}$ and $\mathrm{N}_{3}=3$ seedling tubers hill $^{-1}$ 
Table 4. Combined effect of seedling tuber size and clump planting on the number of leaves hill ${ }^{-1}$ and leaf area index at different days after planting

\begin{tabular}{ccccccccc}
\hline \multirow{2}{*}{ Treatments } & \multicolumn{9}{c}{ Number of leaves plant $^{-1}$} \\
\cline { 2 - 9 } & $40 \mathrm{DAP}$ & $55 \mathrm{DAP}$ & $70 \mathrm{DAP}$ & $100 \mathrm{DAP}$ & $40 \mathrm{DAP}$ & $55 \mathrm{DAP}$ & $70 \mathrm{DAP}$ & $100 \mathrm{DAP}$ \\
\hline $\mathrm{S}_{1} \mathrm{~N}_{1}$ & $28.20 \mathrm{~g}$ & $34.77 \mathrm{~g}$ & $51.80 \mathrm{fg}$ & $63.30 \mathrm{~h}$ & 2.32 & 2.21 & $3.89 \mathrm{efg}$ & $4.45 \mathrm{e}$ \\
$\mathrm{S}_{1} \mathrm{~N}_{2}$ & $27.07 \mathrm{~g}$ & $35.20 \mathrm{~g}$ & $49.23 \mathrm{~h}$ & $61.90 \mathrm{~h}$ & 2.35 & 2.59 & $3.61 \mathrm{~g}$ & $4.04 \mathrm{f}$ \\
$\mathrm{S}_{1} \mathrm{~N}_{3}$ & $29.10 \mathrm{~g}$ & $39.00 \mathrm{f}$ & $49.73 \mathrm{gh}$ & $63.33 \mathrm{~h}$ & 2.60 & 3.06 & $3.82 \mathrm{fg}$ & $4.40 \mathrm{ef}$ \\
$\mathrm{S}_{2} \mathrm{~N}_{1}$ & $32.73 \mathrm{f}$ & $40.97 \mathrm{f}$ & $53.77 \mathrm{f}$ & $67.03 \mathrm{~g}$ & 2.81 & 3.04 & $3.89 \mathrm{efg}$ & $4.48 \mathrm{e}$ \\
$\mathrm{S}_{2} \mathrm{~N}_{2}$ & $34.83 \mathrm{f}$ & $45.20 \mathrm{e}$ & $56.40 \mathrm{e}$ & $67.67 \mathrm{~g}$ & 2.82 & 3.12 & $4.06 \mathrm{ef}$ & $4.44 \mathrm{ef}$ \\
$\mathrm{S}_{2} \mathrm{~N}_{3}$ & $37.73 \mathrm{e}$ & $47.57 \mathrm{de}$ & $59.13 \mathrm{~d}$ & $70.17 \mathrm{f}$ & 3.06 & 3.29 & $4.24 \mathrm{e}$ & $4.69 \mathrm{e}$ \\
$\mathrm{S}_{3} \mathrm{~N}_{1}$ & $40.50 \mathrm{~d}$ & $46.70 \mathrm{e}$ & $58.38 \mathrm{de}$ & $73.07 \mathrm{e}$ & 3.35 & 3.62 & $4.63 \mathrm{~d}$ & $5.14 \mathrm{~d}$ \\
$\mathrm{~S}_{3} \mathrm{~N}_{2}$ & $42.93 \mathrm{~cd}$ & $50.90 \mathrm{c}$ & $62.93 \mathrm{c}$ & $74.03 \mathrm{e}$ & 3.58 & 3.98 & $4.93 \mathrm{~d}$ & $5.31 \mathrm{~d}$ \\
$\mathrm{~S}_{3} \mathrm{~N}_{3}$ & $40.77 \mathrm{~d}$ & $49.67 \mathrm{~cd}$ & $63.90 \mathrm{c}$ & $76.33 \mathrm{~d}$ & 3.91 & 4.39 & $5.35 \mathrm{c}$ & $5.90 \mathrm{bc}$ \\
$\mathrm{S}_{4} \mathrm{~N}_{1}$ & $44.80 \mathrm{bc}$ & $51.80 \mathrm{c}$ & $66.23 \mathrm{~b}$ & $79.03 \mathrm{c}$ & 3.94 & 4.41 & $5.32 \mathrm{c}$ & $5.81 \mathrm{c}$ \\
$\mathrm{S}_{4} \mathrm{~N}_{2}$ & $45.63 \mathrm{~b}$ & $55.47 \mathrm{~b}$ & $68.23 \mathrm{~b}$ & $82.90 \mathrm{~b}$ & 4.11 & 4.75 & $5.89 \mathrm{~b}$ & $6.22 \mathrm{~b}$ \\
$\mathrm{~S}_{4} \mathrm{~N}_{3}$ & $50.17 \mathrm{a}$ & $59.77 \mathrm{a}$ & $72.87 \mathrm{a}$ & $87.97 \mathrm{a}$ & 4.41 & 5.25 & $6.33 \mathrm{a}$ & $6.64 \mathrm{a}$ \\
\hline $\mathrm{LSD}_{(0.05)}$ & 2.64 & 2.90 & 2.28 & 2.07 & 0.19 & 0.87 & 0.39 & 0.41 \\
\hline
\end{tabular}

In a column, means followed by same letter (s) do not differ significantly at $5 \%$ level of probability, $\mathrm{S}_{1}=6-<7 \mathrm{~g}$, $\mathrm{S}_{2}=7-<8 \mathrm{~g}, \mathrm{~S}_{3}=8-<9 \mathrm{~g}, \mathrm{~S}_{4}=9-\leq 10 \mathrm{~g}, \mathrm{~N}_{1}=1$ seedling tuber hill ${ }^{-1}, \mathrm{~N}_{2}=2$ seedling tubers hill ${ }^{-1}$ and $\mathrm{N}_{3}=3$ seedling tubers hill $^{-1}$

Table 5. Effect of seedling tuber size and clump planting on the fresh and dry mass production plant ${ }^{-1}$ at different days after planting (DAP)

\begin{tabular}{|c|c|c|c|c|c|c|c|c|}
\hline \multirow{2}{*}{ Treatments } & \multicolumn{4}{|c|}{ Fresh mass production $\left(\mathrm{g}_{\text {plant }}{ }^{-1}\right)$} & \multicolumn{4}{|c|}{ Dry mass production $\left(\mathrm{g} \mathrm{plant}^{-1}\right)$} \\
\hline & 40 DAP & 55 DAP & 70 DAP & 100 DAP & 40 DAP & 55 DAP & 70 DAP & 100 DAP \\
\hline \multicolumn{9}{|c|}{ Seedling tuber size } \\
\hline $\mathrm{S}_{1}$ & $46.22 \mathrm{~d}$ & $98.78 \mathrm{~d}$ & $157.5 \mathrm{~d}$ & $233.2 \mathrm{~d}$ & $9.911 \mathrm{~d}$ & $20.71 \mathrm{~d}$ & $32.71 \mathrm{~d}$ & $49.74 \mathrm{~d}$ \\
\hline $\mathrm{S}_{2}$ & $97.62 \mathrm{c}$ & $132.2 \mathrm{c}$ & $190.0 \mathrm{c}$ & $264.4 \mathrm{c}$ & $19.32 \mathrm{c}$ & $27.60 \mathrm{c}$ & $38.77 \mathrm{c}$ & $55.29 \mathrm{c}$ \\
\hline $\mathrm{S}_{3}$ & $135.9 \mathrm{~b}$ & $190.6 \mathrm{~b}$ & $242.8 \mathrm{~b}$ & $314.9 \mathrm{~b}$ & $24.65 \mathrm{~b}$ & $36.23 \mathrm{~b}$ & $46.40 \mathrm{~b}$ & $63.50 \mathrm{~b}$ \\
\hline $\mathrm{S}_{4}$ & $212.5 \mathrm{a}$ & $268.3 \mathrm{a}$ & $337.5 \mathrm{a}$ & $456.0 \mathrm{a}$ & $39.19 \mathrm{a}$ & $49.55 \mathrm{a}$ & $66.17 \mathrm{a}$ & $90.23 \mathrm{a}$ \\
\hline $\operatorname{LSD}_{(0.05)}$ & 4.12 & 5.32 & 4.91 & 8.08 & 1.61 & 1.83 & 2.61 & 2.82 \\
\hline \multicolumn{9}{|c|}{ Seedling tuber hill' ${ }^{-1}$} \\
\hline $\mathrm{N}_{1}$ & $103.9 \mathrm{c}$ & $151.8 \mathrm{c}$ & $211.5 \mathrm{c}$ & $284.9 \mathrm{c}$ & $19.52 \mathrm{c}$ & $29.73 \mathrm{c}$ & $41.41 \mathrm{c}$ & $58.79 \mathrm{c}$ \\
\hline $\mathrm{N}_{2}$ & $120.6 \mathrm{~b}$ & $168.8 \mathrm{~b}$ & $226.1 \mathrm{~b}$ & $308.4 \mathrm{~b}$ & $22.72 b$ & $32.65 \mathrm{~b}$ & $45.43 \mathrm{~b}$ & $63.65 \mathrm{~b}$ \\
\hline $\mathrm{N}_{3}$ & $144.8 \mathrm{a}$ & $196.9 \mathrm{a}$ & $258.3 \mathrm{a}$ & $358.0 \mathrm{a}$ & $27.55 \mathrm{a}$ & $38.18 \mathrm{a}$ & $51.20 \mathrm{a}$ & $71.63 \mathrm{a}$ \\
\hline $\operatorname{LSD}_{(0.05)}$ & 3.63 & 4.61 & 4.25 & 6.99 & 1.39 & 1.59 & 2.26 & 2.44 \\
\hline
\end{tabular}

In a column, means followed by same letter (s) do not differ significantly at $5 \%$ level of probability, $\mathrm{S}_{1}=6-<7 \mathrm{~g}$, $\mathrm{S}_{2}=7-<8 \mathrm{~g}, \mathrm{~S}_{3}=8-<9 \mathrm{~g}, \mathrm{~S}_{4}=9-\leq 10 \mathrm{~g}, \mathrm{~N}_{1}=1$ seedling tuber hill ${ }^{-1}, \mathrm{~N}_{2}=2$ seedling tubers hill ${ }^{-1}$ and $\mathrm{N}_{3}=3$ seedling tubers hill ${ }^{-1}$ 
Table 6. Combined effect of seedling tuber size and clump planting on the fresh and dry mass production plant $^{-1}$ at different days after planting (DAP)

\begin{tabular}{ccccccccc}
\hline \multirow{2}{*}{ Treatments } & \multicolumn{3}{c}{ Fresh mass production $\left(\mathrm{g}\right.$ plant $\left.{ }^{-1}\right)$} & \multicolumn{3}{c}{ Dry mass production $\left(\mathrm{g}\right.$ plant $\left.{ }^{-1}\right)$} \\
\cline { 2 - 9 } & $40 \mathrm{DAP}$ & $55 \mathrm{DAP}$ & $70 \mathrm{DAP}$ & $100 \mathrm{DAP}$ & $40 \mathrm{DAP}$ & $55 \mathrm{DAP}$ & $70 \mathrm{DAP}$ & $100 \mathrm{DAP}$ \\
\hline $\mathrm{S}_{1} \mathrm{~N}_{1}$ & $33.17 \mathrm{j}$ & $85.73 \mathrm{j}$ & $150.80 \mathrm{j}$ & $220.30 \mathrm{i}$ & 6.96 & $17.66 \mathrm{~h}$ & $30.82 \mathrm{i}$ & $47.71 \mathrm{~g}$ \\
$\mathrm{~S}_{1} \mathrm{~N}_{2}$ & $49.33 \mathrm{i}$ & $107.10 \mathrm{i}$ & $163.90 \mathrm{i}$ & $245.30 \mathrm{gh}$ & 10.44 & $22.31 \mathrm{~g}$ & $33.47 \mathrm{hi}$ & $50.59 \mathrm{fg}$ \\
$\mathrm{S}_{1} \mathrm{~N}_{3}$ & $56.17 \mathrm{i}$ & $103.50 \mathrm{i}$ & $157.80 \mathrm{ij}$ & $234.00 \mathrm{hi}$ & 12.33 & $22.16 \mathrm{~g}$ & $33.85 \mathrm{hi}$ & $50.91 \mathrm{fg}$ \\
$\mathrm{S}_{2} \mathrm{~N}_{1}$ & $78.83 \mathrm{~h}$ & $121.30 \mathrm{~h}$ & $182.20 \mathrm{~h}$ & $248.50 \mathrm{fg}$ & 15.62 & $25.57 \mathrm{f}$ & $35.71 \mathrm{gh}$ & $52.49 \mathrm{efg}$ \\
$\mathrm{S}_{2} \mathrm{~N}_{2}$ & $94.67 \mathrm{~g}$ & $127.90 \mathrm{~h}$ & $182.10 \mathrm{~h}$ & $259.40 \mathrm{f}$ & 19.36 & $26.40 \mathrm{f}$ & $38.56 \mathrm{fg}$ & $55.19 \mathrm{def}$ \\
$\mathrm{S}_{2} \mathrm{~N}_{3}$ & $119.40 \mathrm{ef}$ & $147.50 \mathrm{~g}$ & $205.70 \mathrm{~g}$ & $285.40 \mathrm{e}$ & 22.97 & $30.82 \mathrm{e}$ & $42.05 \mathrm{ef}$ & $58.17 \mathrm{~d}$ \\
$\mathrm{~S}_{3} \mathrm{~N}_{1}$ & $114.20 \mathrm{f}$ & $162.50 \mathrm{f}$ & $217.10 \mathrm{f}$ & $282.70 \mathrm{e}$ & 20.92 & $32.25 \mathrm{de}$ & $40.92 \mathrm{ef}$ & $56.50 \mathrm{de}$ \\
$\mathrm{S}_{3} \mathrm{~N}_{2}$ & $126.50 \mathrm{e}$ & $185.10 \mathrm{e}$ & $229.80 \mathrm{e}$ & $290.20 \mathrm{e}$ & 23.41 & $35.13 \mathrm{~d}$ & $45.29 \mathrm{e}$ & $59.17 \mathrm{~d}$ \\
$\mathrm{~S}_{3} \mathrm{~N}_{3}$ & $167.20 \mathrm{~d}$ & $224.20 \mathrm{~d}$ & $281.60 \mathrm{~d}$ & $371.70 \mathrm{~d}$ & 29.61 & $41.31 \mathrm{c}$ & $52.99 \mathrm{~d}$ & $74.82 \mathrm{c}$ \\
$\mathrm{S}_{4} \mathrm{~N}_{1}$ & $189.30 \mathrm{c}$ & $237.60 \mathrm{c}$ & $295.70 \mathrm{c}$ & $388.20 \mathrm{c}$ & 34.58 & $43.43 \mathrm{c}$ & $58.18 \mathrm{c}$ & $78.45 \mathrm{c}$ \\
$\mathrm{S}_{4} \mathrm{~N}_{2}$ & $211.90 \mathrm{~b}$ & $255.10 \mathrm{~b}$ & $328.50 \mathrm{~b}$ & $438.70 \mathrm{~b}$ & 37.68 & $46.77 \mathrm{~b}$ & $64.40 \mathrm{~b}$ & $89.65 \mathrm{~b}$ \\
$\mathrm{~S}_{4} \mathrm{~N}_{3}$ & $236.30 \mathrm{a}$ & $312.30 \mathrm{a}$ & $388.10 \mathrm{a}$ & $541.00 \mathrm{a}$ & 45.30 & $58.44 \mathrm{a}$ & $75.92 \mathrm{a}$ & $102.60 \mathrm{a}$ \\
\hline $\mathrm{LSD}_{(0.05)}$ & 7.25 & 9.22 & 8.51 & 13.99 & 2.79 & 3.17 & 4.52 & 4.89 \\
\hline
\end{tabular}

In a column, means followed by same letter (s) do not differ significantly at $5 \%$ level of probability, $\mathrm{S}_{1}=6-<7 \mathrm{~g}$, $\mathrm{S}_{2}=7-<8 \mathrm{~g}, \mathrm{~S}_{3}=8-<9 \mathrm{~g}, \mathrm{~S}_{4}=9-\leq 10 \mathrm{~g}, \mathrm{~N}_{1}=1$ seedling tuber hill $^{-1}, \mathrm{~N}_{2}=2$ seedling tubers hill ${ }^{-1}$ and $\mathrm{N}_{3}=3$ seedling tubers hill ${ }^{-1}$

Total fresh mass plant $^{-1}$ increased with increasing seedling tuber size. Fresh mass production plant $^{-1}$ was influenced significantly by clump planting. The highest fresh mass plant ${ }^{-1}$ at 40, 55, 70 and 100 DAP were recorded in $\mathrm{N}_{3}$. The lowest fresh mass plant $^{-1}$ at all dates of observations was recorded in $\mathrm{N}_{1}$ (Table 5).

Total fresh mass plant $^{-1}$ increased with increasing number of seedling tuber hill $^{-1}$ that increased the number of stem plant ${ }^{-1}$. There was significant variation among the treatment combinations of seedling tubers size and clump planting. The highest fresh mass plant ${ }^{-1}$ at all dates of observations was recorded in $\mathrm{S}_{4} \mathrm{~N}_{3}$ while the lowest fresh mass plant $^{-1}$ was recorded in $\mathrm{S}_{1} \mathrm{~N}_{1}$ (Table 6).

\subsection{Dry mass production per plant}

Dry mass production plant $^{-1}$ showed significant variation due to the seedling tuber size. The highest dry mass plant ${ }^{-1}$ at 40, 55, 70 and 100 DAP were recorded in $\mathrm{S}_{4}$. The lowest dry mass plant $^{-1}$ at all dates of observations was recorded in $\mathrm{S}_{1}$ (Table 5). Total dry mass was higher in larger tubers because larger seedling tuber had huge stored food material than smaller ones and that promoted increased vegetative growth of the plants. This result is consistent with those of other workers (Reust, 2002; Tohin, 2010) in potato.

Total dry mass plant $^{-1}$ was influenced significantly at all dates of observations due to the clump planting. Maximum dry mass plant ${ }^{-1}$ at all dates of observations was recorded in $\mathrm{N}_{3}$ while the minimum in $\mathrm{N}_{1}$ (Table 5). There was significant variation among the treatment combinations of seedling tubers size and clump planting. The highest dry mass plant ${ }^{-1}$ at all dates of observations was recorded in $\mathrm{S}_{4} \mathrm{~N}_{3}$ (Table 6). 
Table 7. Effect of seedling tuber size and clump planting on dry mass production, crop growth rate, tubers weight and gross yield of potato)

\begin{tabular}{|c|c|c|c|c|c|c|c|c|c|}
\hline \multirow{3}{*}{ Treatments } & \multicolumn{4}{|c|}{ Dry mass production $\left(\mathrm{g} \mathrm{m}^{-2}\right)$} & \multicolumn{3}{|c|}{ Crop growth rate $\left(\mathrm{g} \mathrm{m}^{-2} \mathrm{~d}^{-1}\right)$} & \multirow{3}{*}{$\begin{array}{l}\text { Tubers } \\
\text { weight } \\
\left(\mathrm{g} \mathrm{hill}^{-1}\right)\end{array}$} & \multirow{3}{*}{$\begin{array}{c}\text { Gross } \\
\text { yield } \\
\left(\mathrm{t} \mathrm{ha}^{-1}\right)\end{array}$} \\
\hline & 40 & 55 & 70 & 100 & $40-55$ & $55-70$ & $70-100$ & & \\
\hline & DAP & DAP & DAP & DAP & DAP & DAP & DAP & & \\
\hline \multicolumn{10}{|c|}{ Seedling tuber size } \\
\hline $\mathrm{S}_{1}$ & $198.2 \mathrm{~d}$ & $414.2 \mathrm{~d}$ & $654.3 \mathrm{~d}$ & $994.8 \mathrm{~d}$ & $14.40 \mathrm{a}$ & $16.00 \mathrm{~b}$ & $11.35 \mathrm{~b}$ & $85.85 \mathrm{~d}$ & $17.17 \mathrm{~d}$ \\
\hline $\mathrm{S}_{2}$ & $386.3 \mathrm{c}$ & $551.9 \mathrm{c}$ & $775.5 \mathrm{c}$ & $1106 \mathrm{c}$ & 11.0 & $14.90 \mathrm{~b}$ & $11.01 \mathrm{~b}$ & 100.3 & $20.06 \mathrm{c}$ \\
\hline $\mathrm{S}_{3}$ & $492.9 \mathrm{~b}$ & $724.6 \mathrm{~b}$ & $928.0 \mathrm{~b}$ & $1270 \mathrm{~b}$ & $15.44 \mathrm{a}$ & $13.57 \mathrm{~b}$ & $11.40 \mathrm{~b}$ & $120.00 \mathrm{~b}$ & $24.00 \mathrm{~b}$ \\
\hline $\mathrm{S}_{4}$ & $783.7 \mathrm{a}$ & $990.9 \mathrm{a}$ & $1323 \mathrm{a}$ & $1805 \mathrm{a}$ & $13.81 \mathrm{a}$ & $22.16 \mathrm{a}$ & $16.04 \mathrm{a}$ & $128.90 \mathrm{a}$ & $25.78 \mathrm{a}$ \\
\hline $\operatorname{LSD}_{(0.05)}$ & 32.22 & 36.66 & 52.21 & 56.43 & 1.911 & 4.305 & 2.220 & 8.52 & 1.70 \\
\hline \multicolumn{10}{|c|}{ Seedling tuber hill' ${ }^{-1}$} \\
\hline $\mathrm{N}_{1}$ & $390.4 \mathrm{c}$ & $594.5 \mathrm{c}$ & $828.2 \mathrm{c}$ & $1176 \mathrm{c}$ & $13.61 \mathrm{a}$ & $15.58 \mathrm{a}$ & $11.59 \mathrm{~b}$ & $98.55 \mathrm{~b}$ & $19.71 \mathrm{~b}$ \\
\hline $\mathrm{N}_{2}$ & $454.4 \mathrm{~b}$ & $653.1 \mathrm{~b}$ & $908.7 \mathrm{~b}$ & $1273 \mathrm{~b}$ & $13.24 \mathrm{a}$ & $17.04 \mathrm{a}$ & $12.14 \mathrm{ab}$ & $103.75 \mathrm{~b}$ & $20.75 b$ \\
\hline $\mathrm{N}_{3}$ & $551.1 \mathrm{a}$ & $763.7 \mathrm{a}$ & $1024 \mathrm{a}$ & $1433 \mathrm{a}$ & $14.17 \mathrm{a}$ & $17.36 \mathrm{a}$ & $13.62 \mathrm{a}$ & $123.95 \mathrm{a}$ & $24.79 \mathrm{a}$ \\
\hline $\mathrm{LSD}_{(0.05)}$ & 27.91 & 31.75 & 45.21 & 48.87 & 1.655 & 3.728 & 1.923 & 7.38 & 1.48 \\
\hline
\end{tabular}

In a column, means followed by same letter (s) do not differ significantly at $5 \%$ level of probability, $\mathrm{S}_{1}=6-<7 \mathrm{~g}$, $\mathrm{S}_{2}=7-<8 \mathrm{~g}, \mathrm{~S}_{3}=8-<9 \mathrm{~g}, \mathrm{~S}_{4}=9-\leq 10 \mathrm{~g}, \mathrm{~N}_{1}=1$ seedling tuber hill ${ }^{-1}, \mathrm{~N}_{2}=2$ seedling tubers hill $^{-1}$ and $\mathrm{N}_{3}=3$ seedling tubers hill ${ }^{-1}$

3.8. Total dry mass production of plant per $\mathrm{m}^{2}$ There was a significant variation in total dry mass (TDM) production of plant $\mathrm{m}^{-2}$ at $40,55,70$ and 100 DAP due to seedling tuber size (Table 7). Dry mass $\mathrm{m}^{-2}$ increased with increasing seedling tuber size. The highest TDM $\mathrm{m}^{-2}$ was recorded in $\mathrm{S}_{4}$. In contrast, the seedling tuber size of $6-<7 \mathrm{~g}$ had the lowest TDM m $\mathrm{m}^{-2}$ at all growth stages. Similar results were reported by Tohin (2010).

The effect of clump planting significantly influenced on TDM production (Table 7). Total TDM production $\mathrm{m}^{-2}$ increased with increasing number of seedling tubers hill $^{-1}$. The highest TDM $\mathrm{m}^{-2}$ at $40,55,70$ and 100 DAP was recorded in $\mathrm{N}_{3}$ and the lowest TDM m${ }^{-2}$ was recorded in $\mathrm{N}_{1}$.

The interaction effect of seedling tuber size and clump planting had significant effect on TDM m ${ }^{-}$ ${ }^{2}$ at 55, 70 and 100 DAP (Table 8). The highest $\mathrm{TDM} \mathrm{m} \mathrm{m}^{-2}$ was recorded in $\mathrm{S}_{4} \mathrm{~N}_{3}$ and the lowest
TDM m ${ }^{-2}$ was recorded in $\mathrm{S}_{1} \mathrm{~N}_{1}$ except at 100 DAP while it was minimum in $\mathrm{S}_{2} \mathrm{~N}_{2}$.

\subsection{Crop growth rate}

Crop growth rate (CGR) was significantly influenced by seedling tuber size (Table 7). CGR increased with increasing seedling tuber size. At 55-70 DAP, CGR was higher in $\mathrm{S}_{3}$ followed by the $\mathrm{S}_{4}$ with same rank. The lowest CGR both at 40-55 and 70-100 DAP was recorded in $\mathrm{S}_{2}$. The CGR was higher in larger seedling tuber which might be due to increased TDM plant ${ }^{-1}$. Similar results were reported by other workers (Divis and Barta, 2001 and Tohin, 2010) in potato. They reported that larger tuber produced higher number of stems plant ${ }^{-1}$, highest crop growth rate and yield compared to small ones. The effect of clump planting on CGR was not influenced significantly (Table 7). The interaction effect of seedling tuber size and clump planting on CGR was significant at 40-55 DAP (Table 8). The highest CGR was recorded in $\mathrm{S}_{4} \mathrm{~N}_{3}$ and the lowest in $\mathrm{S}_{2} \mathrm{~N}_{2}$. 
Table 8. Combined effect of seedling tuber size and clump planting on dry mass production,crop growth rate, tubers weight and gross yield of potato

\begin{tabular}{cccccccccc}
\hline \multirow{2}{*}{$\begin{array}{c}\text { Treatme } \\
\text { nts }\end{array}$} & \multicolumn{3}{c}{ Dry mass production $\left(\mathrm{g} \mathrm{m}^{-2}\right)$} & \multicolumn{2}{c}{ Crop growth rate $\left(\mathrm{g} \mathrm{m}^{-2} \mathrm{~d}^{-1}\right)$} & $\begin{array}{c}\text { Tubers } \\
\text { weight }\end{array}$ & $\begin{array}{c}\text { Gross } \\
\text { yield }\end{array}$ \\
& DAP & DAP & DAP & DAP & DAP & DAP & DAP & $\left(\mathrm{g} \mathrm{hill}^{-1}\right)$ & $\begin{array}{c}\text { Dienc } \\
\left(\mathrm{t} \mathrm{ha}^{-1}\right)\end{array}$ \\
\hline $\mathrm{S}_{1} \mathrm{~N}_{1}$ & 139.10 & $353.10 \mathrm{~h}$ & $616.40 \mathrm{i}$ & $1176.00 \mathrm{bc}$ & $14.27 \mathrm{abc}$ & 17.55 & 11.26 & $64.15 \mathrm{f}$ & $12.83 \mathrm{f}$ \\
$\mathrm{S}_{1} \mathrm{~N}_{2}$ & 208.90 & $446.30 \mathrm{~g}$ & $669.50 \mathrm{hi}$ & $1273.00 \mathrm{~b}$ & $15.83 \mathrm{ab}$ & 14.88 & 11.41 & $71.65 \mathrm{f}$ & $14.33 \mathrm{f}$ \\
$\mathrm{S}_{1} \mathrm{~N}_{3}$ & 246.70 & $443.30 \mathrm{~g}$ & $677.00 \mathrm{hi}$ & $1433.00 \mathrm{a}$ & $13.11 \mathrm{bcd}$ & 15.58 & 11.37 & $121.65 \mathrm{bc}$ & $24.33 \mathrm{bc}$ \\
$\mathrm{S}_{2} \mathrm{~N}_{1}$ & 312.50 & $511.30 \mathrm{f}$ & $714.30 \mathrm{gh}$ & $1016.00 \mathrm{e}$ & $13.26 \mathrm{bcd}$ & 13.53 & 11.19 & $103.35 \mathrm{de}$ & $20.67 \mathrm{de}$ \\
$\mathrm{S}_{2} \mathrm{~N}_{2}$ & 387.20 & $528.00 \mathrm{f}$ & $771.30 \mathrm{fg}$ & $1012.00 \mathrm{e}$ & $9.39 \mathrm{e}$ & 16.22 & 11.08 & $90.00 \mathrm{e}$ & $18.00 \mathrm{e}$ \\
$\mathrm{S}_{2} \mathrm{~N}_{3}$ & 459.30 & $616.40 \mathrm{e}$ & $840.90 \mathrm{ef}$ & $1018.00 \mathrm{e}$ & $10.47 \mathrm{de}$ & 14.97 & 10.75 & $107.50 \mathrm{~cd}$ & $21.50 \mathrm{~cd}$ \\
$\mathrm{~S}_{3} \mathrm{~N}_{1}$ & 418.40 & $644.90 \mathrm{de}$ & $818.40 \mathrm{ef}$ & $1050.00 \mathrm{de}$ & $15.10 \mathrm{abc}$ & 11.57 & 10.39 & $98.35 \mathrm{de}$ & $19.67 \mathrm{de}$ \\
$\mathrm{S}_{3} \mathrm{~N}_{2}$ & 468.10 & $702.60 \mathrm{~d}$ & $905.90 \mathrm{e}$ & $1104.00 \mathrm{c}-\mathrm{e}$ & $15.63 \mathrm{ab}$ & 13.55 & 9.26 & $133.35 \mathrm{ab}$ & $26.67 \mathrm{ab}$ \\
$\mathrm{S}_{3} \mathrm{~N}_{3}$ & 592.30 & $826.20 \mathrm{c}$ & $1060.00 \mathrm{~d}$ & $1163.00 \mathrm{c}$ & $15.59 \mathrm{ab}$ & 15.58 & 14.55 & $128.35 \mathrm{ab}$ & $25.67 \mathrm{ab}$ \\
$\mathrm{S}_{4} \mathrm{~N}_{1}$ & 691.50 & $868.60 \mathrm{c}$ & $1164.00 \mathrm{c}$ & $1130.00 \mathrm{~cd}$ & $11.80 \mathrm{cde}$ & 19.67 & 13.51 & $128.35 \mathrm{ab}$ & $25.67 \mathrm{ab}$ \\
$\mathrm{S}_{4} \mathrm{~N}_{2}$ & 753.50 & $935.50 \mathrm{~b}$ & $1288.00 \mathrm{~b}$ & $1183.00 \mathrm{bc}$ & $12.13 \mathrm{cde}$ & 23.51 & 16.83 & $120.00 \mathrm{bc}$ & $24.00 \mathrm{bc}$ \\
$\mathrm{S}_{4} \mathrm{~N}_{3}$ & 906.10 & $1169.00 \mathrm{a}$ & $1518.00 \mathrm{a}$ & $1496.00 \mathrm{a}$ & $17.51 \mathrm{a}$ & 23.31 & 17.79 & $138.35 \mathrm{a}$ & $27.67 \mathrm{a}$ \\
\hline $\mathrm{LSD}_{(0.05)}$ & 55.81 & 63.49 & 90.42 & 97.73 & 3.31 & 7.46 & 3.85 & 14.75 & 2.95 \\
\hline
\end{tabular}

In a column, means followed by same letter (s) do not differ significantly at $5 \%$ level of probability, $\mathrm{S}_{1}=6-<7 \mathrm{~g}$, $\mathrm{S}_{2}=7-<8 \mathrm{~g}, \mathrm{~S}_{3}=8-<9 \mathrm{~g}, \mathrm{~S}_{4}=9-\leq 10 \mathrm{~g}, \mathrm{~N}_{1}=1$ seedling tuber hill ${ }^{-1}, \mathrm{~N}_{2}=2$ seedling tubers hill $^{-1}$ and $\mathrm{N}_{3}=3$ seedling tubers hill ${ }^{-1}$

\subsection{Tuber weight per hill}

The effect of seedling tuber size on tuber weight hill $^{-1}$ was significant (Table 7). Tuber weight hill ${ }^{1}$ increased with increasing seedling tuber size. The highest tuber weight hill $^{-1}$ was recorded in $\mathrm{S}_{4}$ where as, the lowest tuber weight hill $^{-1}$ was recorded in $S_{1}$. The lesser tuber weight in smaller sized seed tuber might be due to fewer tubers hill $^{-1}$ and smaller weight tuber. This result is supported by several workers (Vermaet al., 2007; Gulluoglu and Aroglu, 2009; Tohin, 2010) who reported that tuber yield decreased with decreasing seed tuber weight.

There was a significant variation in tuber weight hill $^{-1}$ due to clump planting (Table 7). Tuber weight decreased with decreasing number of seedling tubers hill ${ }^{-1}$ is the highest tuber weight hill $^{-1}$ being recorded in $\mathrm{N}_{3}$. The interaction effect of seedling tuber size and clump planting on tuber weight hill ${ }^{-1}$ was significant (Table 8 ). The highest tuber weight hill $^{-1}$ was recorded in
$\mathrm{S}_{4} \mathrm{~N}_{3}$ and it was statistically similar to $\mathrm{S}_{3} \mathrm{~N}_{2}, \mathrm{~S}_{3} \mathrm{~N}_{3}$ and $\mathrm{S}_{4} \mathrm{~N}_{1}$. The lowest tuber weight hill ${ }^{-1}$ was recorded in $\mathrm{S}_{1} \mathrm{~N}_{1}$ which was statistically similar to $\mathrm{S}_{1} \mathrm{~N}_{2}$.

\subsection{Gross yield of tuber}

The gross tuber yield was significantly influenced by seedling tuber size (Table 7). Gross tuber yield increased with increasing seedling tuber size. The highest gross tuber yield was recorded in $\mathrm{S}_{4}$. In contrast, the lowest gross tuber yield was recorded in $S_{1}$. The gross tuber yield was lower in smaller seedling tuber size because of producing minimum tuber weight hill 1. The yield of total tuber also increased gradually with increasing seedling tuber size. This result is supported by many workers (Shingrup et al., 2003; Hossain, 2004; Sonawane and Dhoble, 2004; Verma et al., 2007; Gulluoglu and Aroglu, 2009; Tohin, 2010).

The clump planting also significantly influenced the gross tuber yield in potato (Table 7). The 
gross tuber yield increased with increasing seedling tubers hill $^{-1}$. The highest gross tuber yield was in $\mathrm{N}_{3}$ and the lowest tuber yield was in $\mathrm{N}_{1}$. The higher tuber yield is always associated with higher plant population. The results are in agreement with the earlier findings of Hossain (2004). Nankar (1990) by planting one, two and three seedling tubers hill $^{-1}$ in inter cropping system; found that 3 seedling tubers hill $^{-1}$ gave the highest yield. Significant variation was found in the treatment combinations of seedling tuber size with clump planting (Table 8). Gross yield of tuber increased with increasing seedling tuber hill $^{-1}$ with the increasing seedling tuber size. The highest gross tuber yield was recorded in $\mathrm{S}_{4} \mathrm{~N}_{3}$ and the lowest yield was recorded in $\mathrm{S}_{1} \mathrm{~N}_{1}$ which was similar to $S_{1} N_{2}$. This result is in agreement with the earlier findings of Hossain (2004).

\section{Conclusions}

The morphological features such as days to first and $80 \%$ emergence, plant height, number of leaves plant $^{-1}$, leaf area index (LAI), fresh mass production plant $^{-1}$, total dry mass (TDM) plant ${ }^{-}$ ${ }^{1}, \mathrm{TDM} \mathrm{m}^{-2}$ and crop growth rate (CGR)were significantly influenced by seedling tuber size and clump planting. Tuber weight hill ${ }^{-1}$ and gross tuber yield were influenced significantly by seedling tuber size and clump planting. Use of True Potato Seed seedling tuber with single number of tuber hill ${ }^{-1}$ was found feasible for potato production.

\section{References}

Divis, J. and Barta, J. 2001. Influence of the seed tuber size on yield and yield parameters in potatoes. Rostlinna vyroba, 47 (6): 271275. [Cited from AGRIS Abst., 2002].

FAO. 1999. Production Year Book. Food and Agriculture Organization of the United Nations. Rome, Italy. 42: 190-193.

Garg, V. K., Singh, S., Raj, D. and Kumar, A. 2000. Effect of physio-agronomical manipulations on the number and yield of seed tubers in breeders seed in the hills.
Potato, global research and development: Proc. of the Global Conf. on Potato, Dec.6-11, 1999. Vol-1. New Delhi, India, 413-418 pp.

Gulluoglu, L. and Aroglu, H. 2009. Effects of seed size and row spacing on growth and yield of early potato in a mediterraneantype environment in Turkey. African Journal of Agricultural Research, 4 (5): 535-541.

Hossain, M. M. 2004. Production and utilization of seedling tubers derived from true potato seed. PhD thesis, Department of Horticulture, BAU, Mymensingh, 202$205 \mathrm{pp}$.

MOA. 2009. Hand Book of Agricultural Statistics. Market Monitoring and Information System, Ministry of Agriculture (MOA), Govt. People's Repub. Bangladesh. 493 p.

Nankar, J. T. 1990. Standardization of TPS agronomy and performance of TPS populations in Maharashtra. (Gaur, P. C. edited) Commercial adoption of true potato seed technology- Prospects and problems. Central Potato Research Institute, Shimla, India. 68-76 pp.

Reust, W. 2002. What about productivity of small seed potato tubers? Revue Suisse- $d^{\prime}$ Agril., 34 (1):5-8. [Cited from AGRIS Abst., 2003].

Roy, T. S., Nishizawa, T. and Ali, M. H. 2005. Studies in the utilization of true potato seeds: Productivity of tubers under subsequent clonal generations. Journal of the Japanese Society for Horticultural Science, 74: 374-380.

Roy, T. S., Nishizawa, T., Islam, M. S., Razzaque, M. A. and Hasanuzzaman, M. 2009. Potentiality of small seedling tuber derived from True Potato Seed (Solanum tuberosum L.) and its economic return as affected by progeny and clump planting. International Journal of Agriculture Environment \& Biotechnology, 2 (4): 385392. 
Shingrup, P.V., Giri, D. G., Jahagirdar, S. W. and Barde, P. R. 2003.Economics of potato (Solanum tuberosum L.) cultivation as influenced by different agro techniques. Journal of Soils and Crops, 13 (2): 291293.

Sonawane, P. D. and Dhoble, M. V. 2004. Effect of spacing and seedling tuber size on potato (Solanumtuberosum) grown through true potato seed. Indian Journal of Agronomy, 49 (3): 194-197.

TCRC. 2004. Annual Report of 2003-04. Tuber Crops Research Centre, Bangladesh Agricultural Research Institute, Gazipur1701. $13 \mathrm{p}$.
Tohin, M. 2010. Effect of seed weight and plant spacing on growth and yield of seedling tuber cv. Bari TPS-1. MS thesis, Dept. of Agronomy, Sher-e-Bangla Agricultural University, Dhaka-1207, 28-69 pp.

Upadhya, M. D., Cabello, R., Falcon, R. and Chujoy, E. 2003. Effects of location and year of production on hybrid true potato seed quality and performance. Acta Horticulturae, 619: 371-374.

Verma, V., Varshney, S. K., Singh, B. and Kumar, A. 2007.Effect of seedling tuberlet size on seed potato yield of TPS varieties in calcareous soils of north Bihar. Annals of Biology, 23 (2): 137-139. 